\title{
产生受激光发射的工作物稹
}

\section{刘頌 豪}

自 1960 年 Maiman $^{[1]}$ 首先实現了紅宝 石的受激光发射以来，在寻找产生受激光发 射的工作物稹及改进器件的結构与性能方 面, 做了許多工作，取得了丰碩的成果。目 前, 实現受激光发射的工作物盾已不下五十 种之多，其中包括气体、液体(有机溶液)和 固体等不同状态和不同結构的物稹; 輸出波 段已从紫外 2542 埃伸展到紅外 35 微米; 实 現了低温和室温的連續操作器件; 紅宝石的 輸出能量已达 500 焦耳, 已制成輸出能量为 113 焦耳的玻璃受激光发射器, 运用光开关 技术，紅宝石的脉冲峑值功率已从 5 仟瓦剧 增至 10 亿酉; 半导体工作物稹的出現, 大大 提高了器件的效率并簡化了整套装置的結 构。此外对各种工作物貭的光㬐、強相干光与 物稹的相互作用等, 也广泛地开展了砰究。

已实現受激光发射的工作物貭，按基原 材料和发光机构可作如下分类:

I气体：（1）单原子惰性气体；（2)混合 气体; (3) 金属蒸气。

II 杂貭激活焱光晶体：（1）讨渡金偊离 子激活焱光晶体; (2) 希土离子激活策光晶 体；（3）鈳系离子激活笑光晶体。

III 杂稹激活榷光玻璃。

IV 有机策光材料: (1) 芳香族有机材 料；（2）希土鳌合物；（3）光敏有机材料;
(4) 产生喇曼相干散射的有机材料。

$\mathrm{V}$ 牛导体：（1）本征牛导体;（2）杂貭 半导体。

VI 其他工作物稹。

其中 II 、III、IV 三类可归属为杂稹激活 凝聚态工作物稹，它們具有类似的发光机 构; 第 VI 类中包括熔石英以及其他暂难进 行分类的材料，如利用气相化学反应来产生 紅外受激发射等。

参考 Schawlow ${ }^{[2]}$, Kiss ${ }^{[3]}$ 和 Yariv 的 建議，科結合气体及牛导体的特点，工作物 貭应滿足下列要求:

1. 有強的发光, 且其中具有高量子效率 的銳綫;

2. 亚稳态寿命尽可能接近輻射寿命，而 終态能級寿命必須短于亚稳态寿命, 从激发 态至亚稳态的能量轉移过程应力求迅速;

3. 尽可能娍小对受激发射的吸收, 这种 吸收相当于縮短光子的寿命并減少亚稳态的 粒子数;

4. 对光票激发的固体工作物稹要求具有 寬而有效的強吸收带且有高的量子效率;

5. 对牛导体工作物貭要求非平衡載流子 有高的浓度和长的寿命, 且能形成諧振腔。

对于四能級机构, 終态能級远离基态是 有利的。此外, 工作物稹必須具有良好的光 
学均匀性及物理化学性能。

本交根据近三年来工作物稹研究中所取 得的成就，对各种工作物稹的特点及其发展 趋向作某些分析，并着重对杂稹激活笑光晶 体和半导体进行討論。

\section{1 气 体}

$\mathrm{Javan}^{[4]}$ 首先在氨一氛混合气体中覌察到 受激光发射現象, 这也是第一个能进行連續 工作的受激光发射器。此后, 对影响氦一氛器 件稳定振薄輸出 ${ }^{[5]}$ 的因素，輸出受激光发射 的特性 ${ }^{[6]}$, 以及能級跃证的性稹等开展了广 泛的硎究工作。目前, 輸出譜綫筧度 $\Delta v / v$ 值 已达 $10^{-11} \sim 10^{-14}$, 頻率重复性达 $4 \times 10^{-8}$, 我 在单原子惰性气体 $\mathrm{He}, \mathrm{Ne}, \mathrm{Ar}, \mathrm{Kr}, \mathrm{Xe}$ ，混 合气体 $\mathrm{He}-\mathrm{Xe}, \mathrm{Ne}-\mathrm{O}_{2}, \mathrm{Ar}-\mathrm{O}_{2}$ 和金属蒸 汽 $\mathrm{Cs}$ 中实現了受激光发射。

1. 气体工作物稹的特点

气体受激光发射器具有高的单色性和高 的光子簡伤度, 且能連續工作等特点。此外, 与笑光晶体相比, 还具有下列特性:

（1）激发电能可直接轉变为光能，而不 必經过电能一光能一晶体发光中心的激发 能一受激发射等多次的能量轉換过程;

（2）选择能級的自由度較大，工作能級 不必是亚稳态, 輔助工作物稹的加入簡单而 有效; 因而有可能在广闊的波段范围內㹸 受激发射;

（3）基本上不受温度的限制，甚至有可 能在远高于常温的条件下工作;

（4）輸入能量的方式更加合理而有效, 存在大能量輸入的可能性且容易实現大几何 形状的工作体。

目前, 較小的輸出功率仍是气体工作物 稹获得广泛应用的主要障碍。
2. 气体工作物稹产生受激光发射的机构 气体工作物稹产生受激光发射也需經历 能量的吸收、轉移与发射等过程，但比固体 簡单。能量的吸收主要依靠放电电子与气体 原子作非弹性磁撞，但也有利用与凝聚态工 作物稹完全相同的光泵激发机构。

对于非单一气体 (如 $\mathrm{He}-\mathrm{Ne}, \mathrm{He}-\mathrm{Xe}$, $\mathrm{Hg}-\mathrm{Zn}, \mathrm{Ne}-\mathrm{O}_{2}, \mathrm{Ar}-\mathrm{O}_{2}$ 等混合气体或混 合蒸汽), 能量从一原子通过碰撞共振轉移 至另一原子或另一分子。

此外还有一种能量轉移是原子 $(B)$ 从分 子 $(A)$ 中分离出来的分子分解。如 $\mathrm{Ne}-\mathrm{O}_{2}$, $\mathrm{Ar}-\mathrm{O}_{2}$ 等混合气体和 $\mathrm{RbI}$ 分子。受激发的 氛或㲷原子与一未激发的氧分子碰撞，后者 被激发到激发态。受激发的氧分子在能量上 是不稳定的，于是分离出一受激发的氧原子 (处于 $3^{3} P_{2}$ 能級)。当此氧原子从 $3^{3} P$ 跃迁至 $3^{3} S$ 能級时产生 $8446 \AA$ 的受激光发射。

\section{3. $\mathrm{He}-\mathrm{Ne}$ 气体受激光发射器}

$\mathrm{He}-\mathrm{Ne}$ 气体的能級机构如图 1 所示。

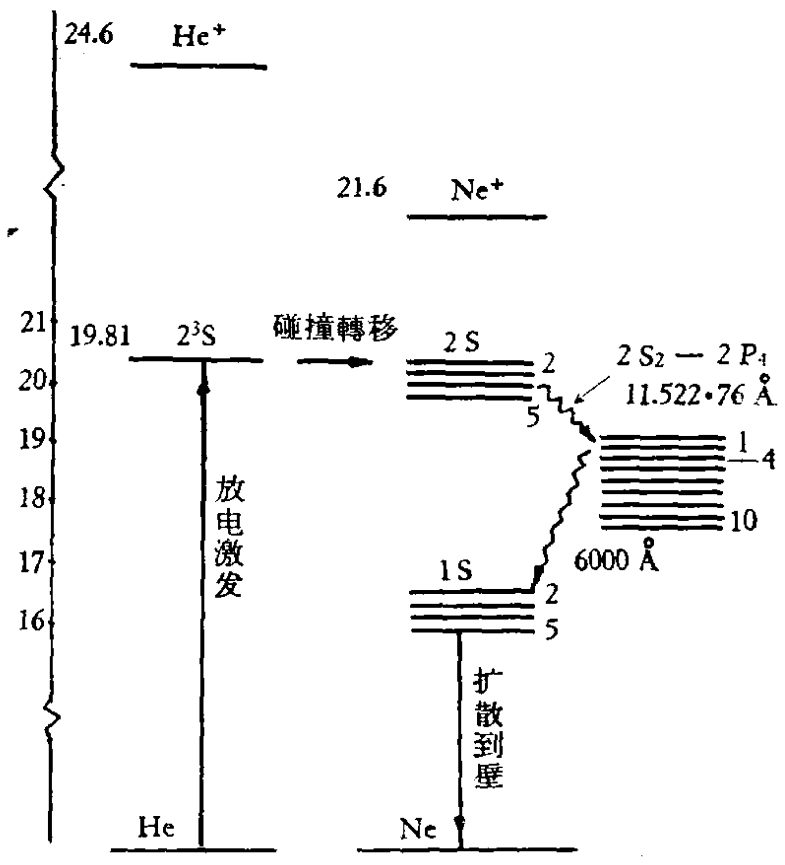

图 1 氦一氞气体的能級机构 
通过射頻放电氦原子被激发到 $2^{3} s$ 亚稳态上。 該态与氛 $2 S$ 态間的能量差很小, 仅为原子热 运动能量的数量級。因此,当处于亚稳态的氦 原子和处于基态的氛原子相磁撞时, 就有可 能使能量轉移給気原子从而使之激发到 $2 S$ 能 級。由于氦、氛間的能量轉移过程非常迅速而 氦原子又有較高的吸收能力，因此能克服其 他过程的能量損耗而容易达到粒子数反轉。

目前对于多能級原子体系的各种光跃迁 几率和非弹性碰撞截面仍缺乏足够的实驗和 理論数据，原子与原子間能量的共振轉移更 是如此。上述問題的研究有利于提高現有气 体受激光发射器的效率和性能, 也有助于定 向寻找寬广波段范围的气体工作物稹。至于 如何提高輸出功率，仍将是今后气体工作物 稹研究的努力方向。

\section{II 杂稹激活熒光晶体}

杂稹激活熒光晶体是实現受激光发射工 作物稹中发展最早、研究范围最广、种类最多 同时也是目前最为成熟的一种。它具有大功 率、大能量輸出以及使用性能稳定等特点。其 基稹晶体可归納为以 $\mathrm{Al}_{2} \mathrm{O}_{3} 、 \mathrm{CaF}_{2}$ 和 $\mathrm{CaWO}_{+}$ 为代表的三种結构类型; 就激活杂稹而論, 可概括为过渡金属离子、希土离子和錒系离 子等三类，其中以希土离子为数最多。已实 現受激光发射的杂稹激活凝聚态工作物稹的 光譜能級，如图 2 所示。今分別討論摻有上 述三类激活杂貭的焱光晶体。

\section{1. 过渡金属离子激活笼光晶体}

过渡金属离子中的 $3 d$ 电子由于沒有外 层电子的屏蔽而直接受晶格場的作用。于 是，在晶体中过渡金属离子的能級位置及性

貭与自由离子的情况有显著不同。

Tanable 和 Sugano[7]在研究金属离子 $d^{n}$

位型的強場矩陣时用 $t_{2}^{N-n} e^{n}(n \leqslant N, n=1$, $2,3,4)$ 来表示位型的能量，式中 $N$ 为电子 的总数, $t_{2}$ 和 $e$ 就一級近似来說可看作是自 由离子 $d$ 軌道的两个量子軌道。在立方晶場 的作用下， $t_{2}$ 軌道的能量将高于 $e$ 的能量。 在含 $d^{n}$ 离子的晶体中可覌察到几个寬的吸 收带和某些类似气体的譜綫。萈吸收带是晶 格热振动的結果，实驗表明郎使在 零度下， 零点振动能仍能产生可覌的寬度。电子間的 庫仑相互作用会引起具有相同电子位型的 $t_{2}^{N-n} e^{n}$ 多重态的分离，它与晶格振动无关。 这是类似气体吸收謭綫的来源。此外，在含 $d^{n}$ 离子的晶体中还观察到焱光光譜。在強立 方場中，銳綫焱光可能是下列能級間跃迁的 結果:

系統 $d^{2} 、 d^{+}$ $d^{3}$ $d^{8}$
跃迁

$$
\begin{aligned}
& { }^{1} E,{ }^{1} T_{2} \rightarrow{ }^{3} T_{1} \\
& { }^{2} E,{ }^{2} T_{1} \rightarrow{ }^{4} A_{2}
\end{aligned}
$$$$
{ }^{1} E \rightarrow{ }^{3} A_{2}
$$

其中 $d^{4}$ 和 $d^{8}$ 在強立方場中上列跃迁确 实是最低激发态的跃迁，但至今仍末发現該 类跃迁的焱光晢綫。 $\mathrm{Al}_{2} \mathrm{O}_{3}: \mathrm{V}^{3+}$ 是 $d^{2}$ 系統的 典型例子，由于晶体具有很強的立方場，可 期望在近紅外区有銳綫焱光光譜，但同样至 今仍未見有这方面的报导。 $d^{3}$ 系統 $\mathrm{Al}_{2} \mathrm{O}_{3}$ : $\mathrm{Mn}^{4+}$ 具有同紅宝石相似的吸收光謭和发光 光謊。虽然 Henkel 对此作了簡单的报导，但 至今仍末实現受激光发射。

在紅宝石中目前已覌察到 $R_{1}, R_{2}, N_{1}$ 和 $N_{2}$ 等四条譜綫的受激光发射現象，此外，还 在 $77^{\circ} \mathrm{K}$ 下实現了連續操作的紅宝石器件。

利用紅宝石所发出的相干光与物稹相互 作用出現了一系列新的物理現象。 Kaiser 和 Garrett ${ }^{[8]}$ 利用紅宝石相干光 $6943 \AA$ 照射 $\mathrm{CaF}_{2}: \mathrm{Eu}^{2+}$ 覌察到双光子效应。也有覌察 


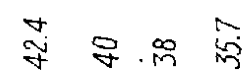
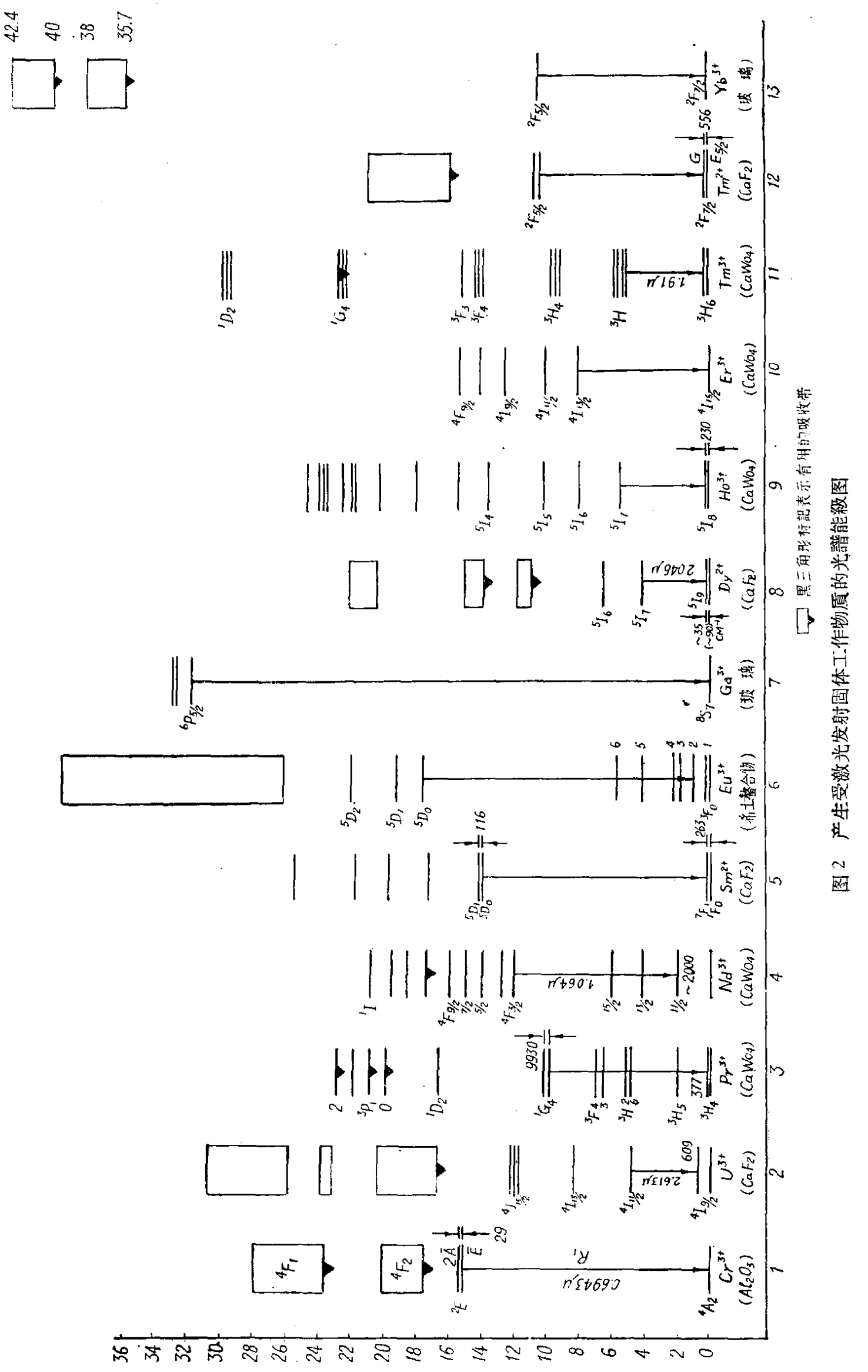
到多环芳香分子晶体中的双光子激发，当， $6943 \AA$ 相下光通过水晶时产生波长为 $3472 \AA$ 的光, 郎结現所謂倍頻效应 ${ }^{[9]}$ 。若与有机溶 液相作用則产生相干喇曼发射。这些現象的 发現及其进一步的䂰究具有极其深刻的意 义，它将有助于相千光用于通訊以及在不同 頻率区域如远紅外区域㹨得相于光源等重大 間題的解决。

看来，为充分发揮过渡金偊离子在受激 光发射研究中的作用，必須寻找合适的基貭 晶体，継續研究过渡金属离子在不同晶格場 们的光譜能級。

\section{2. 希土离子激活策光晶体}

McClure, Dicke, Ельяшевич ${ }^{[10]}$ 对希土离 子光晢进行了归紬、分析和大量的实驗工 作。由于 $5 s$ 和 $5 p$ 外层电子对 $4 f$ 电子的㙚 蔽作用使在不闰介屓中的希土离子光譜与白 由离子光譜非常近似。于是 $4 f$ 能級間跃正 所产生的謭綫具有很小的寬度，且这些跃迁 常是輻射跃迁。如所周知, 大部分希上离子 无論在固体中或溶液中均能发光。在固体工 作物稹中除 $\mathrm{Ce}, \mathrm{Pm}$ 和 $\mathrm{Lu}$ 外其余 11 种希土 离子激活的材料均已实現受激发射，看来这 手不是偶然的。三价希士离子由于外层电子 的屏蔽作用，吸收的振子強度較小，二价希 土离子由于存在 $4 f-5 d$ 能級間的跃迁而具 有較強的吸收。

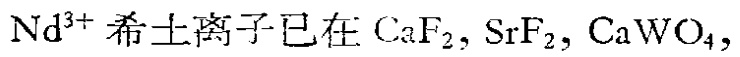
$\mathrm{SrMoO}_{4}$ 及玻璃中笑得紅外受激发射。其中 $\mathrm{CaWO}_{4}: \mathrm{Nd}^{3+}$ 是目前唯一能在室温下得到連 續操作的工作物稹。 $\mathrm{Nd}^{3+}$ 希土离子的紅外 一发光来源于- ${ }^{4} F_{3 / 2} \rightarrow{ }^{4} I$ 多重态的跃正, 最強 者为 ${ }^{4} F_{3 / 2} \rightarrow{ }^{4} I_{11 / 2}$, 位于 1.065 微米。終态能 級在基态上 2000 厘米 $^{-1}$ 处。因此甚至在室 温下, 終态能級仍几乎是空的, 这就无需很
高的激发功率便可点生振藻。 $\mathrm{CaWO}_{4}: \mathrm{Nd}^{3+}$ 的有效吸收带位于 17,000 厘米 ${ }^{-1}$, 連續操作 的輸出功率已达 0.5 无, 在 $\mathrm{CaWO}_{4}$ 中加入适 量的 $\mathrm{Na}$ 离子可簡化电荷补偿机构, 从而使 $\mathrm{CaWO}_{4}: \mathrm{Nd}^{3+}$ 的光譜結构簡单, 效率提高。

$\mathrm{CaF}_{2}: \mathrm{Dy}^{2+}$ 的紅外受激发射来源于 ${ }^{5} I_{7} \rightarrow$ ${ }^{5} I_{8}$ 能級間的跃迁。終态能級位于基态上 90 医米 ${ }^{-1}$ 处。产生受激发射的有效吸收带来自 $4 f-5 d$ 跃迁。亚稳态的寿命长达 10 20 毫 秒, 在 $77^{\circ} \mathrm{K}$ 下可得輸出功率大于 1 瓦的連 續受激发射。在相同的条件下連續操作的闘 值功率为 100 无, 这是目前固体工作物稹中 激发功率最低的一种。据报导利用 50 瓦的 太阳輻射器郎可使 $\mathrm{CaF}_{2}: \mathrm{Dy}^{2+}$ 器件运轉。因 此有可能利用太阳能使卫星上的受激发射装 置进行工作。傎得注意的是二价希士离子目 前仅在 $\mathrm{CaF}_{2}$ 晶体中实現受激光发射，而二 价希土离子的㺅得在工艺上和机构上都是非 常复杂的。利用高能輻射有可能使晶体中的 三价稀土离子还原(或部分还原)为二价稀上 离子, $\mathrm{Sm}^{2+}, \mathrm{Dy}^{2+}$ 和 $\mathrm{Tm}^{2+}$ 郎为一例, 但二价 离子不太稳定往往容易裉色。晶体經受高能 輻射作用后除可能改变其中杂稹离子的价态 外，还可能形成色心及其他陷阱中心，从而 使光譜能級及发光机构复杂化。Browns ${ }^{[11]}$ 曾 提出 $\mathrm{CaF}_{2}: \mathrm{Sm}^{2+}$ 的发光与晶佮中色心的能量 轉移有关。Феофилов ${ }^{[12]}$ 在研究希土激活 $\mathrm{CaF}_{2}$ 晶体的光譜能級时发現希士离子的价态有循 环改变的規律。

$$
\begin{aligned}
& \mathrm{Eu}^{2+}+\mathrm{Sm}^{3+} \stackrel{\text { 短波輻射輻炤 }}{\longrightarrow} \mathrm{Eu}^{3+}+\mathrm{Sm}^{2+} \\
& \mathrm{Eu}^{3+-}+\mathrm{Sm}^{2+} \stackrel{400-600{ }^{\circ} \mathrm{C}}{\underset{\text { 加 热 }}{\longrightarrow}} \mathrm{Eu}^{2+}+\mathrm{Sm}^{3+}
\end{aligned}
$$

看来运用敏化机构改变离子的价态及电 荷补偿方式, 以提高晶体的吸收能力和簡化 发光光譜結构, 可能是扩大工作物盾品种和 
提高工作物稹效能的重要途径。

\section{3. 錒系离子激活熒光晶体}

鋏系离子与鑭系希土离子相似，5f电子 受外层 $6 s$ 和 $6 p$ 电子所屏蔽，但 $5 f$ 軌道的 半径比 $4 f$ 軌道大, 外层电子的屏蔽作用不 如希士离子，也就是說 $5 f$ 电子与晶格偶合 比 $4 f$ 电子強, 因此晶体中錒系离子的光譜 能級与自由离子有較显著的差別, 且比希士 离子有更強的吸收能力。鈳系元素大部分具 有放射性，其中釷和鈾是天然的长时間同位 素，其他都是放射性活泼的元素。Cunningham, Gruen, Conway 等䂰究了鈳系离子 $\mathrm{Pu}^{3+}$ $\mathrm{Am}^{3+}, \mathrm{Np}^{3+}, \mathrm{Cm}^{3+}$ 等在 $\mathrm{LaCl}_{3}$ 晶体中的笑光 光譄, $\Phi$ Фефилов ${ }^{[13]}$ 首先㸴究了 $\mathrm{CaF}_{2}: \mathrm{U}^{3+}$ 的光 譜能級。継紅宝石之后, Sorokin 和 Stevenson 在 $\mathrm{CaF}_{2}: \mathrm{U}^{3+}$ 晶体中覌察到紅外受激发射現 象。 $\mathrm{CaF}_{2}: \mathrm{U}^{3+}$ 属四能級机构, 2.613 微米受 激发射起源于 ${ }^{4} I_{11 / 2}-{ }^{4} I_{9 / 2}$ 能級間的跃迁, 終态能級离基态 609 厘米 ${ }^{-1}$, 在 $4.2^{\circ}-77^{\circ} \mathrm{K}$ 温度范围內亚稳态能級的寿命約 $130 \pm 15$ 微 秒。利用这种能級特点 Boyd 等制成 $\mathrm{CaF}_{2}$ : $\mathrm{U}^{3+}$ 的連續操作器件。Yariv 还在 $\mathrm{SrF}_{2}: \mathrm{U}^{3+}$ 和 $\mathrm{BaF}_{2}: \mathrm{U}^{+}$晶体中覌察到脉冲受激发射关发現 $\mathrm{CaF}: \mathrm{U}^{3+} 2.24$ 微米的相干輸出。Yariv 訩为 随着晶体中三角对称与立方对称比例的改 变, 輸出相干輻射可自 2.24 微米变为 2.613 微米。最近 Willke ${ }^{[14]}$ 等发現 $\mathrm{CaF}_{2}: \mathrm{U}^{3+}$ 的光 譜与 $\mathrm{U}^{3+}$ 离子浓度有着密切关系。当 $\mathrm{U}^{3+}$ 离 子浓度低于 $0.0005 \%$ 时发射光譜相当簡单， 达 $0.007 \%$ 时郎非常复杂, 并訩为 2.57 微米 与2.61 微米的相干輻射是由于高浓度抮杂的 結果。因此目前在 $\mathrm{CaF}: \mathrm{U}^{3+}$ 中最低限度有 2.61 微米 $\left(3830\right.$ 厘米 $\left.{ }^{-1}\right), 2.57$ 微米 $\left(3890\right.$ 厘米 $\left.^{-1}\right)$, 2.51 微米 (3884 厘米 ${ }^{-1}$ ), 和 2.24 微米 (4470 厘米 $\left.^{-1}\right)$ 四条笑光譜綫可产生相干振蕩。

\section{III 杂貭激活焱光玻璃}

由于希土离子外层电子的良好屏蔽作 用，在晶体中能产生受激发射的希土离子同 样也有可能在玻璃中得到，其中特別是稳定 的三价希士离子。利用 $\mathrm{Nd}^{3+}$ 激活鋇 冤玻璃 已实現受激光发射，且已制成长 450 毫米、 直径約 6 毫米、輸出能量达 113 焦耳的器 件。值得特別提出的是紫外受激发射首先在 $\mathrm{Gd}^{3+}$ 激活鋁鎂硅酸盐玻璃中得到。此外，还 首先在玻璃态工作物稹中覌察到 $\mathrm{Yb}^{3+}$ 的受激 光发射現象。

杂貭激活熒光玻璃和熒光晶体的发光机 构基本上相同。仅由于玻璃和晶体在結构上 的差別,前者形成极不对称的晶格場，从而对 杂稹离子的光譄能級带来不同的影响。玻璃 工作物稹的特点在于样品的几何尺寸和形状 不受限制，可制成大尺寸的长棒、玻璃絲或 其他形状的工作体，能得到良好的光学均与 性，在制备工艺上較簡单，成本也較低廉。 此外，在玻璃中可加入比在晶体中数量更多 的激活离子，便于研究基稹对各种激活中心 的影响，且由于能形成玻璃的系統很多，基 稹可在較广泛范围內改变，有利于研究基稹 对发光中心的影响。与晶体工作物 稹 相比, 笑光玻璃的发光譄綫較弥散，产生受激光发 射的閾值功率較高。

为扩大熒光玻璃的应用，应进一步研究 玻璃介稹中希土离子光譜弥散的本貭。选择 具有良好性能特別是高热导率和热稳定性的 玻璃介稹拜运用敏化机构，可作为提高現有 玻璃工作物貭性能的重要途径。研究希士、 个別鈴系和过渡金属离子在不同成分玻璃介 稹中的价态及其光譜能級对增加玻璃工作物 稹的品种具有重要意义。 


\section{IV 有机螢光材料}

自紅宝石实現受激光发射后，利用有机 焱光材料来产生受激发射的建議相 継提出。 1961 年 11 月 Morantz 等利用芳香烴分子, 首先在有机熒光材料中覌察到受激发射現 象。这是由芳香烴分子中 $\pi$ 电子的跃证来实 現的。此后 Morantz, Dalan 和 Schimitschek 分別提出实現受激发射的各种不同机构。特 別值得提出的是 Eckhardt, Heilewarth 等观 察到有机材料的受激喇曼散射現象。这一現 象的发現将为強光与物稹相互作用的非綫性 效应研究开辟道路。

有机焱光材料虽已实現受激发射, 但仍 未見有实际应用的报导。由于有机材料結构 和性貭的多样性, 其产生受激发射的机构在 現有工作物貭中是最多的一种, 且有机材料 既可生长成单晶, 也可制成玻璃和各种溶液 （如水溶液、有机极性溶液和非极性溶液等）。 上述特性对液体工作物稹的获得, 并对能量 轉移、基貭与发光中心的作用以及物貭結构 与产生受激发射的关联等問題的了解可能是 非常有利的。在各种有机策光材料中, 希土 螯合物具有更为广闊的发展前途。

\section{1. 芳香族有机化合物}

这一类型的工作物貭主要依賴有机分子 的自吸收和自发射或利用分子間的能量轉移 机构来实現受激光发射。它属分立中心发光 机构, 同时也具有钽化发光的特点。

\section{2. 希士鳌合物}

Schimitschek 利用苯酰丙酮一銪首先在希 土鳌合物中实現了受激光发射。Weissman ${ }^{[15]}$ 等早已报导某些希土 $(\mathrm{Eu}, \mathrm{Sm}, \mathrm{Tb})$ 螯合物的 发光机构 (图 3)。設 $S$ 和 $T$ 分別为有机配位 体的单重态和三重态能級。 $R$ 和 $F$ 为希土离
子的共振能級和基态能級。与前述机构相类 同，鳌合物通过单重态吸收能量，在 $S^{\prime}$ 的 部分电子以短暫时間跃迁回基态同时发出焱 光，也可以非輻射跃迁至三重态的亚稳能級 上。 $T$ 能級上的电子或发射磷光而回到基态 或通过非輻射跃迁至希土离子的共振能級 $R$ 上，从 $R$ 可发射表征希土 $4 f$ 跃迁的綫状发 光。如 $R$ 和 $F$ 間能造成粒子数反轉則可产生 受激光发射。利用此机构可弥补三价希土离 子吸收能力較弱的缺点。选择合适的有机配 位体有可能得到量子效率較高的希土鳌合物 的工作物稹。

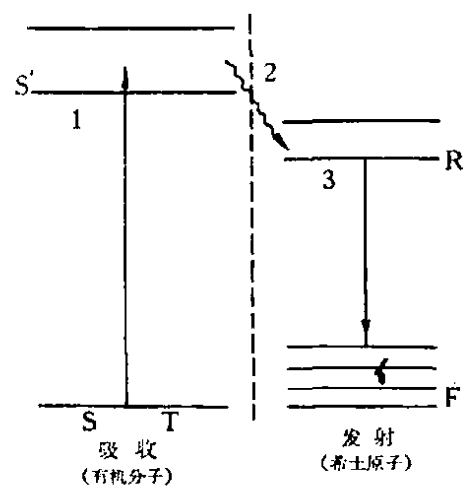

图 3 希土螯合物能量轉移机构

\section{3. 光敏有机化合物}

Dalan 等在 $N N N^{\prime} N^{\prime}$-四甲基对苯二胺 中覌察到受激光发射現象。产生受激发射可 能包括电子的光氧化电离, 电子的迁移、受 陷 (或俘获), 热或光的再激发以及复合等过 程。这种机构显然与半导体的复合发光机构 有相同之处。詳細情况佾有待进一步研究。

\section{4. 产生喇曼相干散射的有机材料}

Eckhardt 等在运用克尔盒光开关进行大 功率紅宝石器件实驗时覌察到 7670 埃的受 激光发射。这是硝基苯克尔盒光开关所发射 的相干喇曼散射現象。 


\section{V 半导体工作物稹}

1962年, Hall, Nathah 和 Holonyak 分別 在 GaAs $p-n$ 結中闰时覌察到脉冲紅外受激发 射。此器件的特点为效率高、装置簡单、可 調頻、在实用上具有广闙的前途。这一成就 是受激发射科学領域发展中的重大进展，为 光学、牛导体物理学与量子电子学的相互渗 透开辟了道路。継上述成就之后又取得了惊 人的进展，实現了液氦温度下的連續操作 ${ }^{[16]}$ 和室温下的脉冲操作 $[17]$ 器件，与此同时 对 GaAs $p^{-n}$ 結的发光机构 ${ }^{[18]}$ 和輸出受激发射 的性稹 ${ }^{[19-20]}$ 正在进行深入的研究。

1. 牛导体工作物稹的特点

与笑光晶体相比,牛导体有下列諸特点:

（1）可利用多种激发方式和激发机构来 注入能量，如光注入、电洼入和高速电子激 发等。利用电注入使电能直接变为光能, 从 而提高器件的效率升簡化装置的結构。

（2）利用能带間跃迋所产生的受激光发 射，其波长一般落在紅外区。由于牛导体能 級結构的多样性，其所能产生的受激发射的 波段范围可能相当寬广。利用本征半导体已 观察到 InSb 5.3 微米的发光，預料利用同一 能带內的能級跃过有可能实現远紅外波段的 受激发射, 这种机构可能成为开拓远紅外領 域楛途径之一。

（3）許多牛导体材料的能带結构及有关 物理性稹均已积累較丰富的資料和数据，有 可能根据成熟的理論对某些材料能否实現受 激光发射作出制断。但有关牛导体发光及輻 射跃迁机构的知傤和实驗数据仍非常缺之。 目前从工艺上已有可能制成純度高、結构完 整和光学均与性良好的半导体单晶，种已掌 握摻杂工艺和器件制备技术。
（4）借控制注入电流密度来改变輸出受 激发射的頻率 ${ }^{[21]}$ 使有可能实現調頻。由于牛 导体的載流子有效稹量小，利用外加磁場也 可能改变发射頻率。半导体具有高的折射率 从而具有高的反射系数, 利用此特性, 牛导 体样品本身郎可作为諧振膑。

（5）能級結构的多样性和复杂性，給斗 导体产生受激光发射带来某些困难，其中最 显著的如自由載流子对激发能量及輸出輻射 的吸收以及通过其他杂稹中心的非輻射复 合，均在不同程度上損耗能量或降低电子一 空资对的寿命，从而提高激发能的閾值，降 低輸出功率甚至于无法实現受激光发射。

为扩展牛导体在受激发射領域的应用范 围和樑入了解在半导体中产生受激发射的机 构，今后必需广泛研究牛导体的光謭及弛豫 机构。为此，牛导体的紅外光譜、紅外发光 以及电子磁共振等方法必須迅速建立。

2. 利用半导体实現受激发射的各种可能 方案

Neuman, Aigrain 和 Tarep ${ }^{[22]}$ 曾提出利 用牛导体来实現超高頻振薄的放大和发生的 建議。其后, Krömer ${ }^{[23]} \mathrm{BacoB}^{[24]} \mathrm{Lax}^{[25]}$ 和 Sugano ${ }^{[26]}$ 等相継提出产生光頻和紅外受激发 射的各种方案。此等方案和建議主要是利用 牛导体中能带之間 (包括导带与滿带或此等 能带与杂貭能級之間）的跃迁和同一能带內 載流子的跃迁来造成粒子数反轉并产生受激 光发射。半导体的堅直跃迁, 非堅值跃迁和 激子复合等均属能带間的跃迁机构, 而利用 具有負有效稹量状态的电子或利用迴旋共振 等則属同一能带內的跃迁机构。目前对上遮 机构均相应屡开研究，其中利用堅直跃迁 $(p-n$ 結) 已覌察到受激光发射現象。

（1）牛导体能带間的堅直跃迁 
由于牛导体中非本衡載流子的弛豫时間 軦短而直接复合的几率又較小, 要实現負温 度必須具备高的激发功率。然而較高功率的 激发, 势必引起牛导体的过热效应从而影响 負温度的获得。为解决此困难可利用脉冲激 发程使少导体于低温下操作。为更容易得到 較高的非平衡載流子浓度释降低激发功率, 选择具有較小禁带寬度和較小載流子有效稹 量的牛导体材料可能是有利的。从上述观点 看, III-V 族半导体材料可能最有希盟。

（2）能带間的非堅直跃迁

$\mathrm{BaCOB}^{[27]}$ 首先提出利用非竪淔跃迁在能 带間产生負温度的建議手誈为此机构产生負 温度所需的載流子浓度比竪直跃迁机构低几 个数量級。已知非堅直跃迁的过程必然伴随 有声子的吸收和发射。若牛导体处于低温 下，則由于晶格振动減弱，再不能提供非 㢣直跃迁吸收过程所需的声子。于是长波声 子的吸收几率必然小于发射几率。因此, 仅 当戴流子的浓度比平衡时稍許增加, 郎有 可能克服此跃迁的吸收而产生受激光发射。 $\mathrm{BaCOB}^{[23]}$ 訩为利用此机构实現負温度必須满 足下列条件:

$$
\omega_{r} / \omega_{f}<T_{\text {有效 }} / T,
$$

式中 $\omega_{r}, \omega_{f}$ 分別为光子和声子的頻率， $T_{\text {有效 }}$ 为与能带間跃迁相应的有效温度, $T$ 为样品 温度。就 $\mathrm{Ge}$ 来說 $\omega_{r} / \omega_{f} \approx 25$, 样品需冷却 到液氦温度才能得到負温度, 而 $\mathrm{Si}, \omega_{r} / \omega_{f} \approx$ 10 , 可在較高温度下得到。利用非堅直跃迁

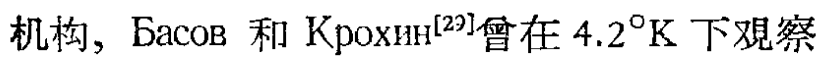
到負温度。Kpoxni ${ }^{[30]}$ 进一步訩为利用非堅直 跃迁机构产生負温度所需的激发功率过高, 如利用激子吸收則有可能降低激发功率。

(3) 激子吸收和复合发光

在某些牛导体中, 如 $\mathrm{Si}^{[31]}, \mathrm{CdS}^{[32]}$ 等已覌
察到激子的綫状发光。Sugano ${ }^{[20]}$ 提出利用激 子发光的尖銳譜綫来实現受激光发射，并䚯 为其有利之处不仅在于存在綫状发光且有极 強的吸收带。Thomas ${ }^{[33]}$ 等研究了利用 CdS 来 产生受激光发射的可能性。由于发光效率低 且需高的激发能量密度及低温, 加以激发深 度太浅很难找到合适的諧振腔机构，因此仍 未得出肯定的結果。他們䚯为关鍵問題在于 提高松料的发光效率。但必須指出, Thomas 等仅考虑必激发的有关問題, 若利用电場激 发或快速电子电离等激发机构則有可能克服 其中某些困难。

Kрохин $^{[30]}$ 和 Dumke ${ }^{[34]}$ 等分析了激子对 非堅㨁跃迁机构产生受激光发射所起的作 用。 Крохин 訩为在非竪直跃迁机构中, 仅当 电子的直接复合几率超过件随有声子吸收的 带內跃迁几率时才能产生負温度, 由于此二 过程均有声子参与, 因此几率値相近。若利 用激子的非㹂直跃过, 則由于形成激子所需 的能量比本征吸收的能量小，且在低温下激 子的复合几率大于自由載流子的复合几率， 这就有可能降低激发功率，也有可能在激子 浓度不高的情况下得到負温度。

能带間的跃迁机构除上述几种方案外， Lax $^{[25]}$, Combrisson ${ }^{[35]}$ 和 Feher ${ }^{[36]}$ 等还提出利 用牛导体杂稹能級的方案。

（4）同一能带的跃迁机构

$\dot{L}^{2}{ }^{[25]}$ 和 Tarep $^{[2]}$ 分別提出利用牛导体 迴旋共振来产生和放大紅外及微波輻射的建 議。已知載流子在靜磁場 $H$ 及交变电場作用 下将沿螺旋形曲綫运动，迴旋頻率为

$$
\omega_{11}= \pm \frac{e H}{m_{ \pm c}^{*}}
$$

* 对于满带的空穴, 存在同样的可能性。 
式中 $m_{ \pm}^{*}$ 分別表示空穴和电子的有效稹 量, 它們按相反的方向旋轉； $c$ 为光速。当交变电 場頻率 $\omega$ 等于迴旋頻率 $\omega_{\text {M }}$ 时, 就可观察到 共振吸收。对于某些具有基本吸收謭綫的牛 导体如 $\mathrm{Ge}$ 和 $\mathrm{Si}$ 等, 当产生迴旋共振吸收时

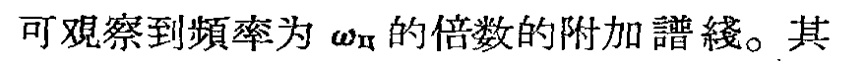
結果导致选择定則 $\Delta n= \pm 1$ 的破坏, 同时 出現异于零值的二偶矩跃迁 $\Delta n= \pm 2.3$ 。这 种現象原則上可用来产生和放大高頻輻射。

假設高頻交变电場的強度足够大, 便能 在热弛豫时間內有足够数量的載流子跃迁到 高能級, 于是在系統中会产生或放大頻率为 $i_{\mathrm{c}}=l \omega_{\mathrm{H}} / n=l \omega_{\text {H }}\left(l=1,2, \cdots, \omega_{\mathrm{H}}=n \omega_{\mathrm{HI}}\right.$, $n=1,2, \cdots)$ 的輻射。若取 $l=1, n=2$, 根据 Tarep 的估計，产生及放大頻率为 $\dot{c}_{\mathrm{e}}=\omega_{\mathrm{Ir}}=\omega_{\mathrm{H}} / 2$ 的輻射, 激活載流子浓度必 須达到 $N_{\text {棌活 }} \approx 3 \hbar / 4 \pi Q_{1} \tau\left|d_{1}\right|^{2}$, 式中 $\left|d_{1}\right|$ 为二 极矩跃迁, $Q_{1}$ 为当 $\omega_{\mathrm{H}}=n \omega_{\mathrm{II}}$ 时, 諧振腔的 品稹因素。在 $N_{\text {治活 }} \approx 10^{10} \mathrm{~cm}^{-3}$ 而 $\omega_{\mathrm{c}}=2 \pi$ $\times 10^{11}$ 赫时, 輻射的最大功率約为 5 毫瓨/厘 米 $^{3}$, 即高于一般分子或順磁振蕩器。

此后, Луговой ${ }^{[37]}$ 呼究了在直流磁場下, 引入一电导张量, 覌察置于其中的半导体介 稹对弱单色輻射的吸收, 发現在迴旋共振頻 率下吸收降至零值。其結果与 Lax 和 Taгер 所估計者不同。Kassel ${ }^{[38]}$ 訩为由于所需的激 发功率过高, 利用迴旋共振方法在牛导体中 很难实現受激发射。当然, 目前要下肯定性 的結論仍末免为时过早。

Krömer ${ }^{[23]}$ 會建議利用半导体中具有負 有效稹量的載流子来实現受激发射。其后， Mattis $^{[39]}$, Kaus ${ }^{[40]}$ 和 $\mathrm{BaCOB}^{[24,28]}$ 等又作了进一 步分析。已知处于导带較低能級的电子其有 效稹量为正，而处于导带較高能級的电子为 負, 如能設法使大部分电子处于負有效盾量
状态，即处于导带的較高能級时，就有可能 产生受激发射。 $\mathrm{BacoB}$ 討論了恆定电場和脉 计电場作用下的情况，訩为在恆定电場下， 无論在有效貭量为各向同性或各向异性的情 况中均不可能形成負温度状态。在脉冲电場 下，虽可望能形成負温度，但載流子寿命非 常短 ( $\approx 10^{-10} \sim 10^{-12}$ 秒) 是实現过程中的主 要障碍。

\section{GaAs $p-n$ 結}

利用 $\mathrm{GaAs}$ 半导体早已制成太阳能电池, 并具有良好的性能。与硅、鍺相比, GaAs 具 有寬的禁带寬度 (GaAs: $1.35-1.51$ 电子伏， $\mathrm{Si}: 1.1, \mathrm{Ge}: 0.65$ ) 和較高的电子正移率 (GaAs: $4000 \sim 8000$ 厘米 ${ }^{2} /$ 伏秒, $\left.\mathrm{Si}: 1200, \mathrm{Ge}: 3900\right)$ 。 这可能是 GaAs $p-n$ 結能在室温下操作与能 产生連續受激发射的主要原因之一。

\section{(1) $\mathrm{GaAs}$ 的复合发光}

早在 1955 年 Braunstein ${ }^{[41]}$ 提出了 GaAs 复合发光的光謭分布測定結果, 并发現复合 輻射相应的能量比禁带寬度小 0.3 电子伏, 可 訩为不是本征复合的发光。Наследов 等測定 了 GaAs 的复合发光的光譜分布, 他們䚯为 长波部分的发光与导带同杂貭能級的复合有 关，而短波部分則属本征复合发光。此外还 发現长波与短波复合发光的強度与通过 $p-n$ 結的电流密度有关。在高电流密度注入的情 况下有可能产生粒子数反轉。Keyes 亦得出 类似的結果郑覌察到在低温下強发射綫的譜 綫寬度变窄。Nathan 在 GaAs $p-n$ 結中覌察 到 1.473 电子伏的窄譜綫（电或光注入）科 一再強調这是导带电子和 $\mathrm{Zn}$ 受主空穴复合 的結果。最近他还发現在 $1.5143 \pm 0.0005$ 电 子伏 $\left(4.2^{\circ} \mathrm{K}\right)$ 处有激子发射。

(2) GaAs $p-n$ 結

已产生受激发射的 GaAs $p-n$ 結一般利 
用摻有浓度約为 $10^{17}$ 厘米 ${ }^{-3} \mathrm{Te}$ 的 $n$ 型 GaAs 作为原材料, 然后扩散入浓度的为 $10^{18} \sim 10^{19}$ 厘米 ${ }^{-3}$ 的受主杂稹 $\mathrm{Zn}$ 而构成 GaAs $p^{-n}$ 結。 根据 $p-n$ 結理論在 $p$ 型和 $n$ 型牛导体的交界 处形成高阻区一势垒。GaAs $p-n$ 結諧振腔的 結构如图 4 所示。

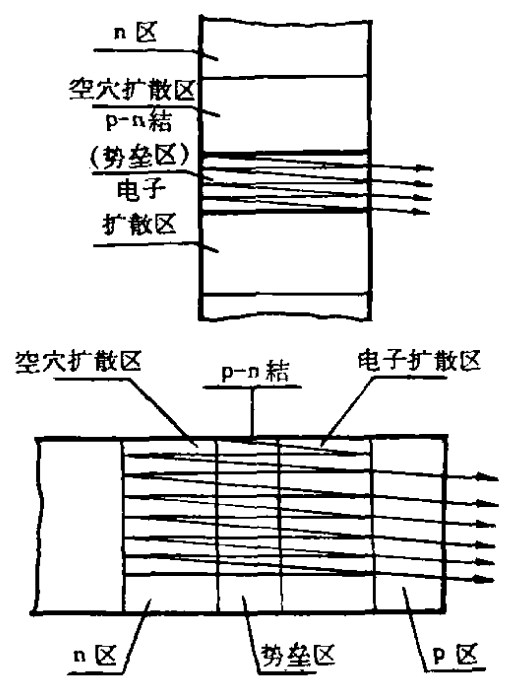

图 4 利用 $p-n$ 結作为諧振腔

$\mathrm{bacoB}^{[42]}$ 指出在牛导体 $p-n$ 結中实現負 温度的条件为: $E_{(F) n}-E_{(F) p}>E_{g}$ 。 要淪 足上述条件必須：（1）高浓度摻杂造成簡并， 郎使施主和受主的費米能級落在导带和满带 之內;（2）更大地降低势垒, 这就必須增 加注入电流的密度和提高非本衡載流子的浓 度。簡科情况下伏一安特性如图 5 所示。在 伏一安曲綫的前段为隧道电流区域相当于㯌 道二极管的特性曲綫。 $\mathrm{BaCoB}$ 訩为产生受激 发射的机构应相当于伏一安曲綫的扩散部分。

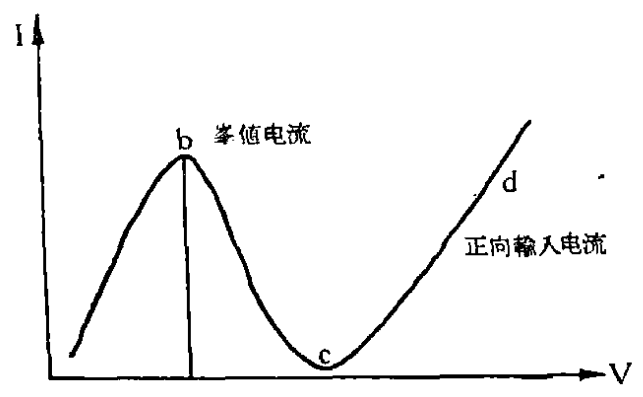

图 $5 p-n$ 結的伏姿特性曲綫
Адирович ${ }^{[43]}$ 和 $\mathrm{GacoB}^{[42]}$ 分析了在 $p^{-n}$ 結 中为得到負温度所必需的注入电子浓度, 科 訩为注入浓度将随牛导体材料簡科程度的增 加和样品温度的降低而減小，如由于高浓度 摻杂而使杂稹能級与满带或导带重迭而形成 混合带并导至載流子有效稹量变小时，則負 温度更容易实現。

最近 Nathan ${ }^{[44]}$ 通过光注入和电注入方 法观察到在 $p$ 区的发光完全相应于导带至受

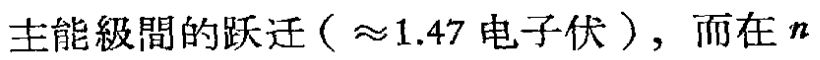
区才覌察到与禁带寬度相应的輻射 $(\approx 1.51$ 电子伏），但前者的輻射強度比后者高两个 数量級。因此可訩为利用通过 Zn 中心的复 合比之在 $n$ 区复合更为有效。

(3) GaAs $p-n$ 結的受激发射效应

已制成产生脉冲受激发射 GaAs $p^{-n}$ 結

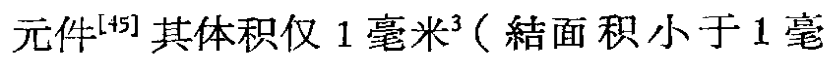
米 $\left.^{2}\right) 。 p-n$ 結的势垒区及自由載流子扩散 长度的范围師构成諧振腔。注入最大脉冲电 流㟳度为 $10^{4}-10^{6}$ 安/厘米 ${ }^{2}$, 脉冲持續时間 約为 $1-5$ 微秒, 在 $77^{\circ} \mathrm{K}$ 下輸出相干輻射 的波长为 8400 埃，譜綫寬度約为 $0.2 \sim 0.3$ 埃，量子效率約 20-50\%。輸出最大功率为 15 瓦。温度降低至 $4^{\circ} \mathrm{K}$, 輸出性能将有所 改进。对于 $\mathrm{Ga}\left(\mathrm{As}_{1-x} \mathrm{P}_{x}\right) p^{-n}$ 結輸出波长为 $6200-8400$ 埃。

产生連續发射的装置 ${ }^{[16]}$ 在液氦下操作， 激发电流䄪为 100 岀/厘米 ${ }^{2}$ 輸出輻射波长为 8400 埃, 但不全为相干輻射。輸出总功率約 10-25 毫无。据报道有可能提高工作温度 和增加輸出功率。

最近 Newman ${ }^{[46]}$ 已将 GaAs $p-n$ 結受激 光发射器用作 $\mathrm{CaWO}_{4}: \mathrm{Nd}^{3+}$ 和 $\mathrm{CaF}_{2}: \mathrm{Dy}^{2+}$ 的 激发源，前者的效率达 $85 \%$, 后者則达 40 $50 \%$ ，从面大大提高了能量轉換效率，簡化 
了装置的結构，乎降低了装置的价格。

\section{VI 工作物盾的发展趋向}

受激光发射在国防上和科学技术上的应 用为工作物稹的发展提供了明确的方向。

1. 发展大能量和大功率脉冲輸出的工作 物貭。为此, 有必要开展下列研究工作:

（1）提供光学均匀性良好和大尺寸的紅 宝石单晶; 研究晶体生长及退火工艺与晶体 缺陷、光学均匀性的閾値和輸出能量关系;

（2）䂰究光学均匀性良好和大尺寸的 $\mathrm{CaWO}_{4}: \mathrm{Nd}^{3+}$ 单晶的生长与退火工艺以及其 它材料如掺 $\mathrm{Nd}^{3+}$ 焱光玻璃的制备, 研究 电荷补偿机构、敏化机构、基盾成分及結构 等因素的影响;

（3）性能和比上述材料优越的新型工作 物稹，呼究某些高熔点、耐高温而又具有高 热导率的材料作为基稹的可能性; 研究提高 工作物貭狜灭温度和工作温度的可能途径;

（4）探索气体、液体和牛导体工作物稹 的提供大能量輸出的可能途径。

在这个問題上,生长高貭量、大尺寸的耐 高温晶体的工艺研究具有特殊重要的意义。

2. 发展大功率連續操作的工作物稹, 开 展下列研究工作:

（1）发展现有气体受激发射的机构，探 索提高輸出功率的可能途径;

（2）进一步提高具有四能級机构塋光晶 体的性能, 例如增強掺希士晶体的吸收能力, 提高工作物稹的工作温度; 为此, 必需研笔 晶体中各种吸收机构、能量轉移过程和弛豫 机构以及高能輻射对掺杂熒光晶体的光譜效 应与电子过程, 其中尤应注意热弛豫問題;

（3）发展連續操作斗导体和有机工作物 貭。
3. 发展适用于远紅外波段的工作物稹， 填补 35-500 微米受激发射的空白区。利用 气体分子和有机分子的轉动能級，牛导体的 杂貭能級或同一能带內能級間的跃迁，或利 用拍頻、混頻技术等有可能实現該波段范围 的受激光发射。目前应着手㸴究利用混頻来 产生远紅外受激发射的途径。此外，还需洼 意实現短波（如紫外、X射綫和 $\gamma$ 射綫）受 激发射的可能途径及其可能应用。

4. 发展非綫性介稹, 梁入㸴究強相干光 与物稹的相互作用。

为开展上述工作，应研究下列具有共同 性的問題:

（1）工作物稹的提純、掺杂及制备。如 属固体工作物稹应着重研究单晶的生长工 艺，生长条件、退火与晶体缺陷及光学均匀 性的关系，激活杂貭的分布和扩散規律以及 新晶体的生长工艺与生长机理。

（2）发光中心的生成及其結构。激活杂 稹梁埋在基貭晶格中而形成所謂发光中心。 为此,必需研究发光中心的几何結构、激活杂 稹与基稹晶体的結构、物理化学性稹及其相 互配置与分布、杂稹离子在基貭中形成不同 价态的可能性、由于不等价置換而引起的电 倚补偿或对称性的变化以及由于高能輻射或 其他外界条件的作用而引起晶格缺陷、色心 更价态的变化等。这里有必要运用光学、电 学、磷共振和物理化学等方法来进行綜合的 研究。

（3）发光体的光譜能級。这里包括吸收 光譜、发光光譜、能級的稳定性及其寿命、 譄綫寬度及其影响因素以及晶格場和外場如 温度磁場、电場、压力对光譄能級的影响。

（4）能量轉移过程及驰豫机构。工作物 稹避过各种吸收机构吸收能量, 然后通过多 
种方式轉移給发光中心。必需研究能量的各 种轉移方式以及能量轉移过程中杂厧离子之 間以及杂貭离子与晶格之間的相互作用，拜 运用有效的方法和工具来研究弛豫过程。

（5）強相干光与物貭的相互作用过程及 其与物稹結构的关系。

上述問題特別是 $2 、 3 、 4$ 三个問題是密 切联系不可分割的。此外基本問題的研究应 密切結合前述四大方面。

本文由刘䅡豪执笔，由沃新能、林达基、林开华、蔡 英时、店武等同志提供素村茾經謨絽汉、邓錫銘、干福喜、 呂大元等同志校閔。在緛写过程中承滕永祿、林青柏、陈 远春、王福貴、高淑兰、刘亚淑、李航孝、傅恩生等闹志 热情帮助,特此志謝。

[1] Maiman T. H., Nature, 187, 493, (1960); Bri. tish Commuications and Electronics, 7, 674, (1960).

[2] Schawlow A. L., JOSA, 51, 472 (1961).

[3] Kiss Z. J. and Duncan R. C. Jr., Proc. IRE, 50, 1531 (1962).

[4] Javan A., et al, Phys. Rev. Letters, 6, 106 (1961).

[5] Paananen R., et al, Proc. IEEE, 51, 63 (1963).

[6] Herriott D. R., JOSA, 52, 1, 31-37 (1962).

[7] Sugano S. and Tanab Y. e, J. Phy. Soc. (Japan), 13, 880 (1958).

[8] Kaiser W. and Garrett C. G. B., Phys. Rev. Lett., 7, No. 6, 229 (1961).

[9] Janable Y., Prog. Theoret. Phys. (Kyoto), Suppl. No. 14, 17 (1960); Sugano S., Prog. Theoret. Phys. (Kyoto), Suppl. No. 14, 66 (1960).

[10] Ельяшевич М. А. Спектры редских земель, Гостехиздат (1953).

[11] Browns P. E., Proc. Phys. Soc., 79, 1085 (1962).

[12] Феофилов П. П., Изв. АН СССР Серия Физическая 26, 435 (1062).

[13] Галкин Л. Н. и Феофнлов П. П., ДАН СССР 114, 745 (1957).

[14] Willke J. P., Kiss Z. J., Duncan R. C., and McCormick J. J., Proc. IEEE, 51, 56 (1963).

[15] Weissman S. I., Chem, Phys. J., 10, 214 (1942).

[16] Electronic News, "Continuous operation of GaAs Laser at IBM", vol. 7, No. 347, Nov. 19, 27 (1962).
[17] Burns G. and Nathan M. I., IBM J. Res. and Dev., 72-73, Jannary (1963).

[18] Nathan M. I., Burns G., Phys. Rev., 129, 125 (1963).

[19] Michel A. E., Walker E. J., Bull. Amer. Phys. Soc., Ser. II., 8, No. 1, 88 (Z9) (1963).

[20] Quist T. M., et al, Bull. Amer. Phys. Soc., Ser. 11, 8, No. 1, 88 (Z7) (1963).

[21] Басов Н. Г., Крохин О. Н., Попов Ю. М., Вестник АН СССР, 3, 61 (1961).

[22] Тагер А. С., Гладун А. Д., ҚЭТФ 35, 808 (1958).

[23] Krömer H., Phys. Rev. 109, 1856 (1958).

[24] Басов Н. Г., и др. ЖЭТФ 40, 1203, (1961); ЖЭТФ, 39, 1001 (1960).

[25] Lax B., Quantum Electronics A Symposium, p. 428 . (1960).

[26] Sugano S., Appl. Optics 1, 3 (1962).

[27] Басов Н. Г., Крохин О. Н., Попов Ю. М., ЖЭТФ 39, 1486 (1960).

[28] Басов Н. Г., и др. УФН, 72, 11-209 (1960).

[29] Басов Н. Г., Крохин О. Н., и др. ЖЭТФ 41, 988 (1961).

[30] Крохин О. Н., ФТТ 4, 822 (1962).

[31] Haynes J. R., Phys. Rev. Lett. 4, 361 (1960).

[32] Thomas D. G., Hoppield J. J., Phys. Rev'. 116, 573 (1959).

[33] Thomas D. G., Hoppield J. J., J. Appl. phys. 33, 3243 (1962).

[34] Dumke W. P., Phys. Rev. 127, 1559 (1962).

[35] Combrisson J., Honig A., and. Townes C. H., C. R. Acad. Sci. 242, 2451 (1956).

[36] Feher G., Gordon J. P., Buehler E., Gers E. A. and Thurmond C. D., Phys. Rev. 109, 221 (1958).

[37] Луговой В. Н., ЖЭТФ 41, 1562 (1961).

[38] Kassel S., Proc. IEEE 51, 216 (1963).

[39] Mattis D. C., Stevenson M. J., Phys Rev. Lett. 3, 18 (1959).

[40] Kaus P. Phys. Rev. Lett. 3, 20 (1959).

[41] Braunstein B., Phys. Rev. 99, 1892 (1955).

[42] Басов Н. Г., Крохин О. Н., ГТопов Ю. М., ЖЭТФ 40, 1878 (1961).

[43] Адирович Э. И., и др. ФТТ, 3, 3339 (1961).

[44] Nathan M. I., Burns G., Appl. Phys. Lett, 1, 89 (1962).

[45] N. Holonyak, Jr., S. F. Bevacqua, App. Phys. Letters, 1, No. 4, 82 (1962).

[46] Newman R., J. Appl. Phys. 34, 437 (1963); Electronic News 8, no. 355, 1. 14. p. 1. (1963). 